九州大学学術情報リポジトリ

Kyushu University Institutional Repository

\title{
SOME SPHINGIDAE COLLECTED IN THAILAND (LEPIDOPTERA)
}

\section{Azuma, Seizi}

Entomological Laboratory, College of Agriculture, University of the Ryukyus

https://doi.org/10.5109/2538

出版情報: ESAKIA. Special Issue 1, pp.155-160, 1990-04-20. Entomological Laboratory, Faculty of Agriculture, Kyushu University バージョン :

権利関係 : 


\title{
SOME SPHINGIDAE COLLECTED IN THAILAND (LEPIDOPTERA)
}

\author{
Seizi Azuma \\ Entomological Laboratory, College of Agriculture,University of the Ryukyus, \\ Nishihara, Okinawa, 903-01 Japan
}

\begin{abstract}
Forty two species of Sphingidae (Lepidoptera) collected in Thailand are enumerated. Of which twenty four species are here recorded for the first time from Thailand. Twenty two species are photographed.
\end{abstract}

\section{Introduction}

Tams, 1924, J. Nat. Hist. Soc. Siam $6: 229-289$, recorded 26 species of the Sphingidae from Thailand. During my Thailand survey in 1979, 1980 and 1981, under the Project of "Ecological studies on shifting cultivation and its transformation process to sustained upland farming", I tried to collect as many sphingid moths as possible. My collecting sites are : the Khon Kaen University Farm at Chulabhon Dam (Nam Phrom Dam), Amphoe Khon San, Changwa Chaiyaphun, is located about 140 $\mathrm{km}$ west of Khon Kaen, a general elevation ca. $800 \mathrm{~m}$. But one specimen was collected at Khon Kaen Province, Northeast Thailand.

Most of the material are preserved in the collection of the Entomological Laboratory, College of Agriculture, University of the Ryukyus, but some are in Dr. H. Inoue's collection.

I wish to express my sincere thanks to Dr. H. Inoue, Otsuma Women's University, for his kind advice and valuable guidance. I also wish to thank Mrs. Juthatat Attajarusit, Div. of Animal and Entomology, Ministry of Agriculture, Thailand and Mr. Sawaeng Ruaysoongnem, College of Agriculture, Khon Kaen University for their helping in field survey.

The species here first recorded from Thailand will be indicated with an asterisk after species name.

\section{List of the Sphingidae collected in Thailand}

\section{Acherontia lachesis (Fabricius)}

19, 7. viii. 1980 .

2. Acherontia styx styx (Westwood)

16, 29. ix. 1981.

3. Agrius convolvuli (Linnaeus)

10̛, 30. ix. 1981; 10 , 7. x. 1981.

4. Meganoton nyctiphanes (Walker)

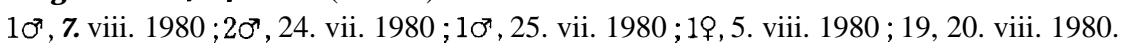


5. Psilogramma menephron menephron (Clamer) 4619, 18. vii. $1980 ; 3 \sigma^{\top} 3$ ㅇ, 19. vii. $1980 ; 4 \sigma^{\top} 1$ \%, 25. vii. $1980 ; 3 \sigma^{\top}$, 18. viii. $1980 ; 5039$, 20. viii. 1980 ; $4 \sigma^{\top}, 26$. viii. $1980 ; 1 \sigma^{\top}, 14$. vii. $1981 ; 19,16$. ix. $1981 ; 10^{\top} 1$ \% , 29. ix. $1981 ; 10^{\top}, 30$. ix. 1981.

6. Psilogramma rufescens rufescens (Butler)*

19, 19. vii. $1980 ; 1$; , 26. viii. 1980.

7. Megacorma oblique (Walker)* 10̛, 20. viii. 1980 ; 19, 17. iv. $1981 ; 2 \sigma^{7}, 18$. vii. 1981.

8. Poliana leucomelas Rothschild \& Jordan* 1, 25. vii. 1980 .

9. Dolbina inexacta (Walker)* $10^{\top}, 18$. vii. $1980 ; 10^{\top}, 19$. vii. $1980 ; 1 \sigma^{\top}, 20$. viii. 1980.

10. Ambulyx ochracea Butler* $1 \sigma^{\top}, 7$. viii. $1980 ; 1 \sigma^{\top}, 8$. viii. $1980 ; 30,18$. viii. $1980 ; 1 \sigma^{\top}, 19$. viii. $1980 ; 5 \sigma^{\top} 2$;, 20 , viii. $1980 ; 1$,



11. Ambulyx substrigilis aglaia (Jordan)* 1ơ, 19. vii. 1980; 20̛', 7. vii. $1980 ; 1$; , 20. viii. 1980.

12. Ambulyx liturata Butler*

1, 14. viii. $1980 ; 1$ ’ , viii. 19. 1980.

13. Ambulyx kuangtungensis (Mell)* 1ơ19, 20. viii. 1980 ; lb, 26. viii. 1980 ; lb, 7. x. 1981.

14. Marumba dyras dyras (Walker) $1 \sigma^{\top}, 18$. vii. $1980 ; 1 \sigma^{\top}, 17$. iv. 1981.

15. Marumba sperchius gigas (Butler)*



16. Parum colligata (Walker)*

19, 15. viii. 1980.

17. Parum porphyria porphyria (Butler)* 1ơ, 19. vii. $1980 ; 1 \sigma^{\top}, 24$. vii. $1980 ; 1 \sigma^{\top}, 19$. viii. 1980 ; lb, 20. viii. $1980 ; 1 \sigma^{\top}, 17$. iv. $1981 ; 1$; 16. ix. 1981 .

18. Cypoides chinensis (Rothschild \& Jordan)* $1 \sigma^{\top}, 7$. viii. $1980 ; 26,8$. viii. $1980 ; 2 \sigma^{\top 1} 1$, 24. viii. $1980 ; 3 \sigma^{\top}, 25$. viii. 1980.

19. Callambulyx poecilus (Rothschild)* $10 ̛$, 25. vii. 1980.

20. Daphnis hypothoushypothous (Cramer) $10^{\top 1} 1$, 18. vii. $1980 ; 1 \sigma^{7}, 19$. vii. $1980 ; 30,25$. vii. $1980 ; 10^{7}, 14$. viii. $1981 ; 361$ \%, 29. ix. $1980 ; 1$, 2.x. $1981 ; 107,7$. x. 1981.

21. Daphnis nerii (Linnaeus) lb, 19. x. 1981, Kohn Kaen.

22. Ampelophaga dolichoides (Felder)* lb, 29. ix. 1981; lb, 25. x. 1981.

23. Acosmeryx Socrates Socrates Boisduval $8 \sigma^{\top} 1$, 18 . vii. $1980 ; 110^{\top 1} 1$, 19. viii. $1980 ; 3 \sigma^{7}, 24$. vii. $1980 ; 26,7$. viii. $1980 ; 16$, viii. $1980 ; 30^{7}$, 19. viii. 1980 ; lb, 25. viii. 1980 ;1 , 26. viii. $1980 ; 10^{7}, 25$. v. 1981 ;26, 7. x. 1981 ; 3o', 17. x. 1981 ; 10̛, 19. x. 1981.

24. Acosmeryx anceus subdentata Rothschild \& Jordan* 16, 18. viii. $1980 ; 2 \sigma^{\top 1} 1$, 19. vii. $1980 ; 2 \sigma^{7}, 8$. viii. $1980 ; 1 \sigma^{\top}, 26$. viii. $1980 ; 1 \sigma^{\top}, 25$. v. 1981(Sawaeng leg.). 
25. Acosmeryx naga (Moore)*

26, 18. viii. 1980.

26. Acosmeryx sericeus sericeus (Walker)*

10', 18. vii. $1980 ; 16,19$. vii. $1980 ; 1 \sigma^{7}, 7$. viii. $1980 ; 2 \sigma^{7}, 8$. viii. 1980.

27. Elibia dolichus (Westwood)'

10’, 7. viii. 1980.

28. Nephele didyma (Fabricius)

10’, 7. viii. 1980.

29. Eurypteryx bhuga (Moore)*

19, 17. iv. 1981.

30. Macroglossum hemichroma (Drury)*

19, 18. ii. 1981.

31. Hippotion boerhaviae (Fabricius)

$10^{7}, 30$. ix. $1981 ; 10^{7}, 7$. x. 1981; 10', 17. x. 1981.

32. Hippotion echeclus Boisduval

1ㅇ, 7. x. 1981.

33. Theretra nessus (Drury)

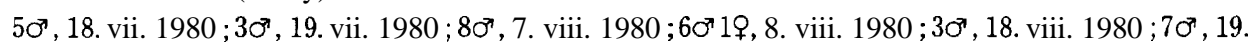
viii. $1980 ; 13 \sigma^{\top}, 20$. viii. $1980 ; 1 \sigma^{\top}, 24$. viii. $1980 ; 4 \sigma^{\top} 1$ ㅇ, 29. ix. $1981 ; 90^{\top 9} 9$, 30. ix. $1981 ; 20^{\top}, 8$. x. 1981; $20^{\prime \prime}, 19$. x. $1981 ; 1$; 2 . xi. 1981.

34. Theretra boisduvalii (Bugnion)*

20 , 7. vii. $1980 ; 10^{\top 1} 1$, 8. vii. $1980 ; 10^{\top 3} 3$, 24. viii. 1980 ; 19, 16. ix, 1981 ;19, 29. ix. $1981 ; 10^{7}, 19$.

x. 1981.

35. Theretra clotho clotho (Drury)

1, 18. vii. $1980 ; 3 \sigma^{\prime} 1$, 19 . vii. $1980 ; 2 \sigma^{\prime}, 25$. vii. $1980 ; 5 \sigma^{\prime} 1$ ㅇ, 7. viii. $1980 ; 1 \mathrm{~b} 1$ \&, 18 . viii. $1980 ; 5$ o , 19. viii. $1980 ; 36,20$. viii. $1980 ; 10^{7}, 17$. iv. $1981 ; 5 \sigma^{\top 1} 1$, 29. ix. 1981; 3ơ 2ㅇ, 30. ix. $1981 ; 20$, 7. x. $1981 ; 10^{\top} 1$ ㅇ, 19. x. 1981.

36. Theretra latreillei lucasii (Walker)

16, 7. viii. 1981; 19, 30. ix. 1981.

37. Theretra lycetus (Cramer)*

10', 7. viii. 1980; 10̛, 18. viii. 1980.

38. Theretra oldenlandiae oldenlandiae (Fabricius)

20, 19. vii. 1980; 20’, 7. x. 1981; 10̛', 8. x. 1981.

39. Theretra pinastrina pinastrina (Martyn)

lb, 7. x. 1981.

40. Pergesa acteus (Cramer)

1\%, 7. viii. $1980 ; 10^{\circ}, 16$. viii. $1980 ; 1$; 24 . viii. $1980 ; 10^{\circ}, 2$. xi. 1981.

41. Cechenena aegrota aegrota (Butler)*

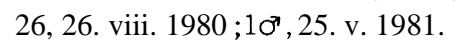

42. Cechenena lineosa Walker'

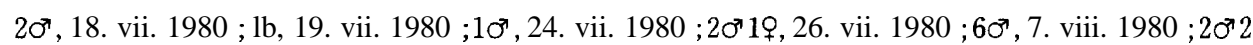



\section{References}

[Department of Agriculture, Royal Thai Government U. S. Okert. Miss. Thailand] 1965. A host list of the insects of Thailand.

Tams, W. H. T., $\quad$ 1924. List of the moths collected in Siam, Jour. Nat. Hist.Soc. Siam, 6(3) : 229-379. 

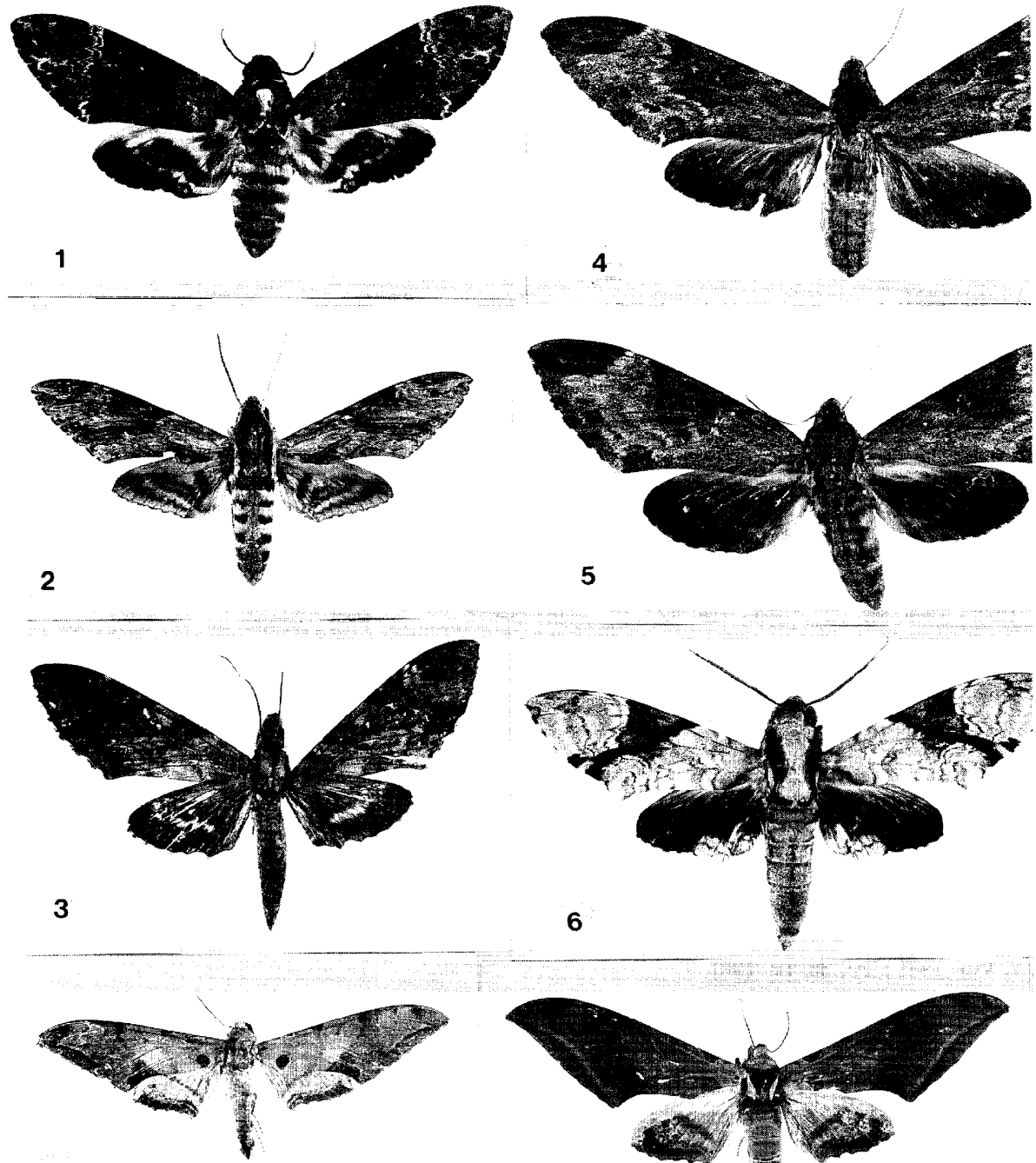

7

8

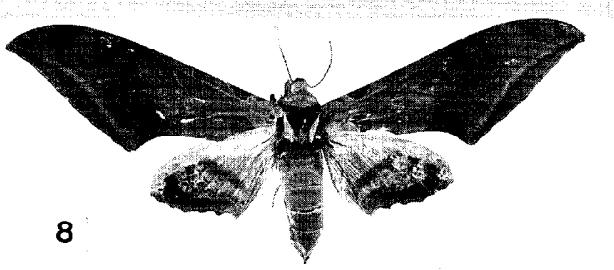

Fig. 1. Acheron tia Zachesis (Fabricius)

Fig. 2. Agrius convolvuli (Linnaeus)

Fig. 3. Dolbina inexacta (Walker)

Fig. 4. Megnoton nyctiphanes (Walker)

Fig. 5. Psilogrammamenephron menephron (Clamer)

Fig. 6. Megacorma oblique (Walker)

Fig. '7. Ambulyx kuangtungensis (Mell)

Fig. 8. Ambulyx ochracea Butler 


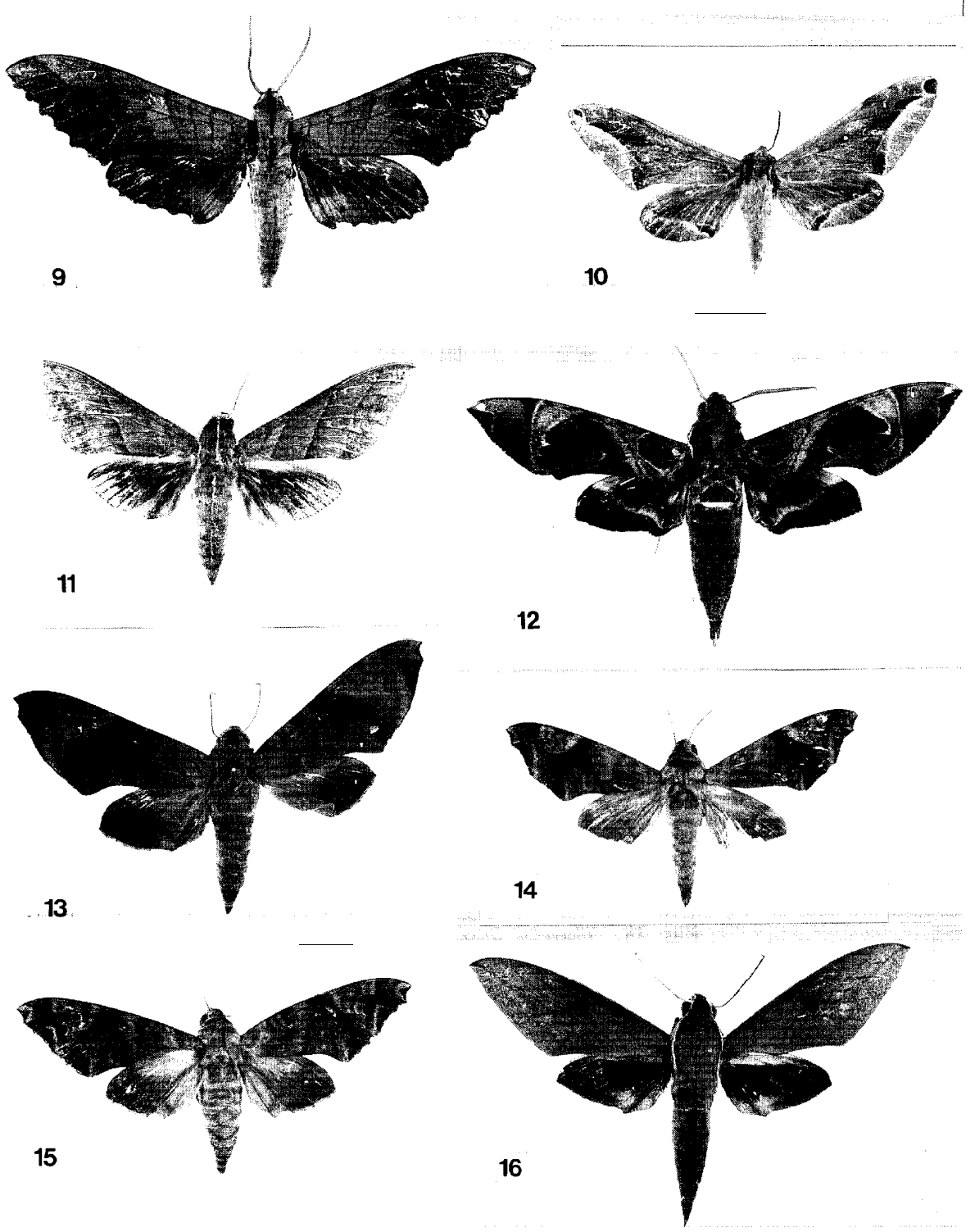

Fig. 9. Marumba sperchius gigas (Butler)

Fig. 10. Parum colligata (Walker)

Fig. 11. Ampelophaga dolichoides (Felder)

Fig. 12. Daphnis hypothous hypothous (Cramer)

Fig. 13. Acosmeryx Socrates Socrates (Boisduval)

Fig. 14. Acosmeryx anceus subdentata Rothschild \& Jordan

Fig. 15. Acosmeryx sericeus sericeus (Walker)

Fig. 16. Theretraboisduvalii (Bugnion) 


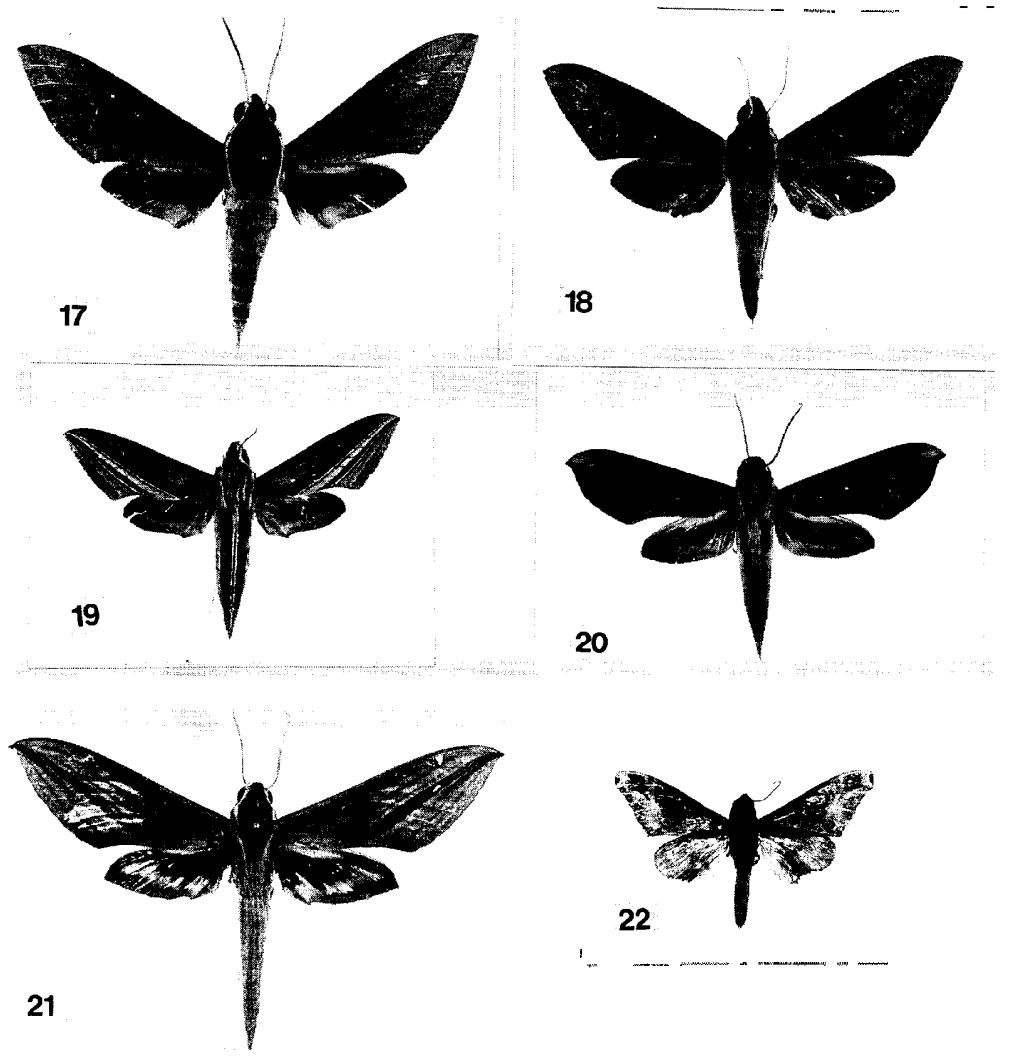

Fig. 17. Theretra clotho clotho (Drury)

Fig. 18. Theretra latreillei lucasii (Walker)

Fig. 19. Theretra oldenlandiae oldenlandiae (Fabricius)

Fig. 20. Pergesa acteus (Cramer)

Fig. 21. Cechenena lineosa Walker

Fig. 22. Parum porphyria porphyria (Butler) 